\title{
Solar activity cycle and rotation of the corona
}

\author{
Z. Mouradian ${ }^{1}$, R. Bocchia ${ }^{2}$, and C. Botton ${ }^{2}$ \\ 1 Observatoire de Paris, LESIA, 92195 Meudon Cedex, France \\ 2 Observatoire de Bordeaux, Avenue Pierre Semirot, 33270 Floirac, France
}

Received 5 June 2002 / Accepted 13 August 2002

\begin{abstract}
In this paper we consider the dependence of the coronal rotation period on solar activity. We analyzed the $10.7 \mathrm{~cm}$ radio emission flux covering cycles 19 to 22 . We have established a new method for studying the rotation rate: by identifying the flux pulses with the recurrence of active longitudes. We first determined the frequency domain of the rotation and then analyzed each cycle separately. The entire domain of 51.7 years was divided into 480-day sections, which were analyzed independently and then grouped into frames for each cycle in order to study the time variation of prominent frequencies (Fig. 4). Power spectra were obtained with the Maximum Entropy Method. The $10.7 \mathrm{~cm}$ emission rotation varies accordingly to the activity level, i.e. at the activity maxima the synodic period of rotation is about 25.4 days and at the minima it is around 30 days; it is 32 days for the quiet Sun (Table 1). Empirical relations give the ratio of maximum and minimum sidereal rotation rate with respect to sunspot number (Fig. 6). During the cycle increase phase, the average acceleration of the rotation period is $0.8^{\circ} / \mathrm{d}$ per year, in decreasing phase that average is only -0.5 .
\end{abstract}

Key words. Sun: activity - Sun: rotation

\section{Introduction}

The rotation of solar atmospheric structures is mainly affected by magnetic tubes rooted in the convective zone. There are two causes of the variation of rotation rates for the various structures: the dynamics of the solar interior (differential rotation, North/South hemisphere asymmetry, depth of anchorage of magnetic ropes and variation of rotation according to solar activity) and local conditions in the atmosphere (magnetic field structure, strength, and height). The review papers of Howard (1984), Schröter (1985), Howard et al. (1991), Libbrecht \& Morrow (1991) and Beck (1999) summarized and analyzed the general aspects of rotation of the solar atmosphere and quoted articles on the target.

The highest precision in rotation measurement is obtained with sunspots. These measurements benefit from more than 100 years of high quality photographic observation. They are generally considered as a reference for the rotation of the solar atmosphere. Numerous authors reported sunspot rotation rates which are in good agreement (Lustig 1983; Gilman \& Howard 1984; Howard et al. 1984; Balthasar et al. 1986; Hathaway \& Wilson 1990; Howard et al. 1999). Some papers approach the problem of sunspot rotation in relation to the solar cycle (Livinston \& Duvall 1979; Lustig 1983; Gilman \& Howard 1984; Balthasar et al. 1986; Pulkkinen \& Tuominen 1998; Gupta et al. 1999) and they conclude that the sun rotates faster during minimum activity. More general results of

Send offprint requests to: Z. Mouradian, e-mail: zadig.mouradian@obspm.fr
Woodard \& Libbrecht (1993) from helioseismology, or Mordvinov \& Plyusinia (2000) from MDI data, confirm the time variation of solar rotation during the cycle of activity. We remind the reader that the Carrington Rotation period was defined by sunspot rotation and it is the reference of solar rotation.

Studies of coronal rotation in relation to the solar cycle and observed at the limb or on the disk, were performed by Hansen et al. (1969), Henze \& Dupree (1973), Antonucci \& Dodero (1977), Fisher \& Sime (1984), Parker (1986), Hoeksema \& Scherrer (1987) and Weber et al. (1999). According to these authors, coronal rotation is characterized by a less pronounced differential rotation than photospheric rotation and a near rigid rotation during minimum activity. We found that the $B$ coefficient (from the differential rotation rule $\Omega^{\star}=A-B \times \sin ^{2} \varphi$ ), which characterizes only the differential rotation, is about 1 for the corona and about $2.5^{\circ} \mathrm{d}$ for sunspots. From Fig. 1 of Hoeksema \& Scherrer, we obtain that the synodic rotation period variation of sunspots and of the corona, between the equator and $30^{\circ}$ latitude, is respectively 1.32 and $0.41 \mathrm{~d}$. This last property made coronal rotation less latitude-dependent throughout the cycle. Sky-Lab observations long ago showed that coronal holes rotate rigidly as well as differentially. From green line data $(\lambda=5305 \AA)$, Badalian \& Livshits (1997) assert that coronal rotation changes during the cycle, whereas Rybak (1994) do not find a clear signature. On the other hand Vats et al. (1999) derive the variation of synodic periods of coronal rotation ranging from 22 to $30 \mathrm{~d}$.

In the present article we analyse corona rotation from $10.7 \mathrm{~cm}$ radio flux. Our aim is to find the dependence of the 


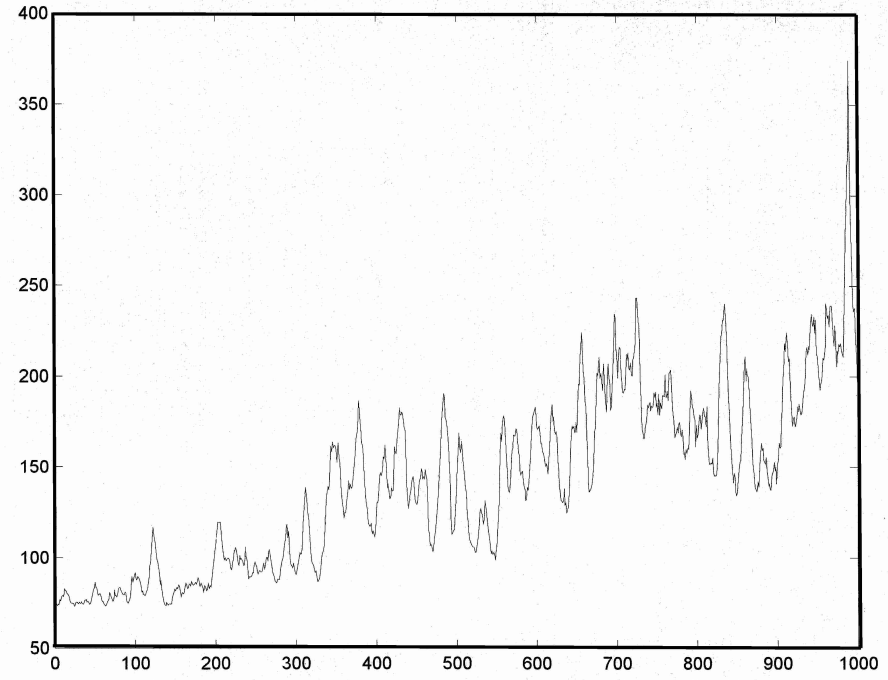

Fig. 1. A thousand-day record of the slowly-varying component of $10.7 \mathrm{~cm}$ radio flux (February 25, 1977 to November 21, 1979). The $y$-axis is the flux, in sfu and the abscissa is in days.

active coronal rotation rate on the $11-y$ solar cycle. We use the day (d) as the unit of synodic rotation period and the degree per day $(\% / d)$ as the sideral rotation rate. Note that, for the latter, case the conversion to other units is $1^{\circ} / \mathrm{d}=0.202 \mu \mathrm{rad} \mathrm{s}^{-1}=$ $32.15 n \mathrm{~Hz}$. In Sect. 2, we display the data we used in this study. In Sect. 3 we briefly give the technique used to measure the rotation rate. In Sect. 4 we show the results and the dependency of the rotation rate on activity cycle. The paper ends with conclusions.

\section{Data}

Our analysis of inner corona rotation related to the solar cycle is based on the radio flux at $10.7 \mathrm{~cm}(2800 \mathrm{MHz})$ monitored by the Dominion Astrophysical Observatory of the National Research Council of Canada (Covington 1969). These observations started in late 1946 and are still continuing. The daily recorded flux is the integrated emission over the solar disk. The quoted flux values are measured around local noon (17:00 UT) and have a relative error of $\pm 2 \%$. Covington mentions that the $10.7 \mathrm{~cm}$ flux is correlated with the active region (sunspots and plages), so that this emission becomes a good index of solar activity. The origin of $2800 \mathrm{MHz}$ radiation is the thermal emission of plasma trapped in magnetic loops of the active region and is consequently related to the photospheric magnetic field flux of active regions. The $10.7 \mathrm{~cm}$ emission is mainly due to free-free radiation, as mentioned by Tapping \& DeTracey (1990). The $10.7 \mathrm{~cm}$ rotation rate concerns the global activity component of the solar corona. The review of Rabin et al. (1991) shows the good correlation of $10.7 \mathrm{~cm}$ flux to solar activity.

The $10.7 \mathrm{~cm}$ emission is located in the low corona. From high-resolution observation of 8 sources Swarup et al. (1963) found that the average altitude of radio emission is $15000 \mathrm{~km}$ above the photosphere. But Kundu (1965) mentioned emission altitudes two or three times higher for some active regions observed by Swarup. Covington (1969) determined even a height

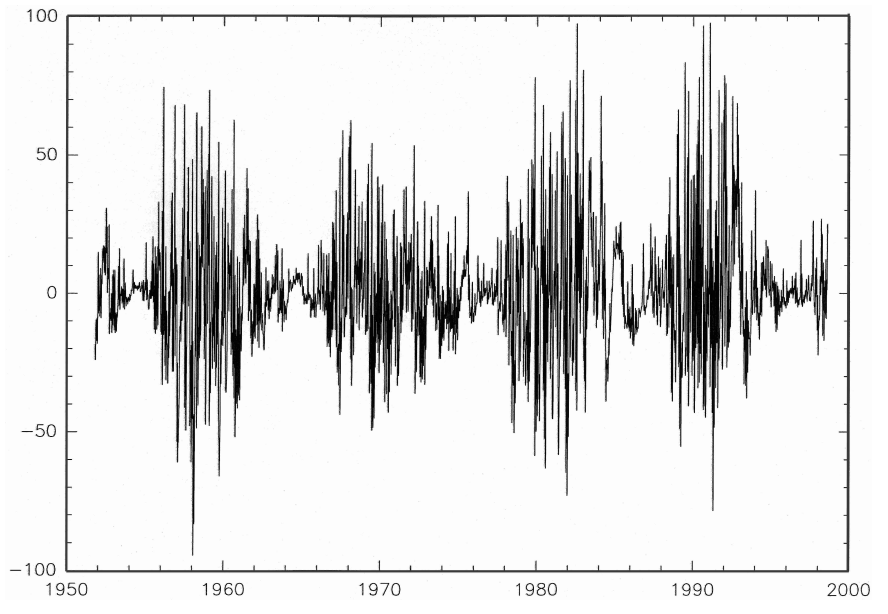

Fig. 2. Radio flux record showing cycles 19 to 22 . The low frequency $(\leq 1 / 365 \mathrm{~d})$ effect is removed.

of 0.2 solar radius, but Vats et al. (2001) gives the altitude as $60000 \mathrm{~km}$. These discrepancies in $10.7 \mathrm{~cm}$ emission height are due to different magnetic complexity and strength of the active regions. Because we use the recurrence method in the present search, the results are independent of the altitude.

The radio flux we use is the Slowly Varying component ( $S$ component) consisting of pulses superimposed on a background. Figure 1 shows a thousand days' recording (February 25,1977 to November 21,1979 ) that cover the growing to maximum phases of cycle 21 . It can be seen that the background intensity is changing during the cycle and is related to the global solar magnetic flux. This is clearly due to the active regions distributed outside of active longitudes, whose number varies with the phase of the activity cycle. For our search we used the "Series D" data, which is adjusted for solar-terrestrial distance variation and corrected to absolute units. The $S$ component is free of bursts of transient events (flares, surges or other).

The modulation of the $S$ component is due to the presence of complex of activity (Gaizauskas et al. 1983) on the solar disk. Van Driel-Gesztelyi et al. (1992) mentioned that more than a third of the active regions are clustered around "active longitudes". SOHO/EIT data analyzed by Benevolenskaya et al. (2000) confirm the reality of active longitudes in the corona. As the life time of the active longitudes is several times longer than the solar rotation period, their successive passages on the solar disk modulate the $10.7 \mathrm{~cm}$ flux and determine the $S$ component. Becker (1955) gives 10 to 12 solar rotations as an average life time for sunspots "hearth" and Brouwer \& Zwaan (1990) give 4 to 6 rotations. The the solar flux is thus modulated by the recurrence of active longitudes, thereby allowing measurement of the rotation rate. Consequently, we introduce a new method for measuring solar rotation rate, based on solar activity rotation with respect to Earth. This way, our method provides solar rotation parametres in absolute units.

The emergence of any new big active region or an active longitude on the solar disk introduces a "perturbation" in the flux modulation, such as those visible in Fig. 1 at day 450, or a longer period around day 700 . The computed power spectra integrate the $480 \mathrm{~d}$ domain, including all disturbances. 


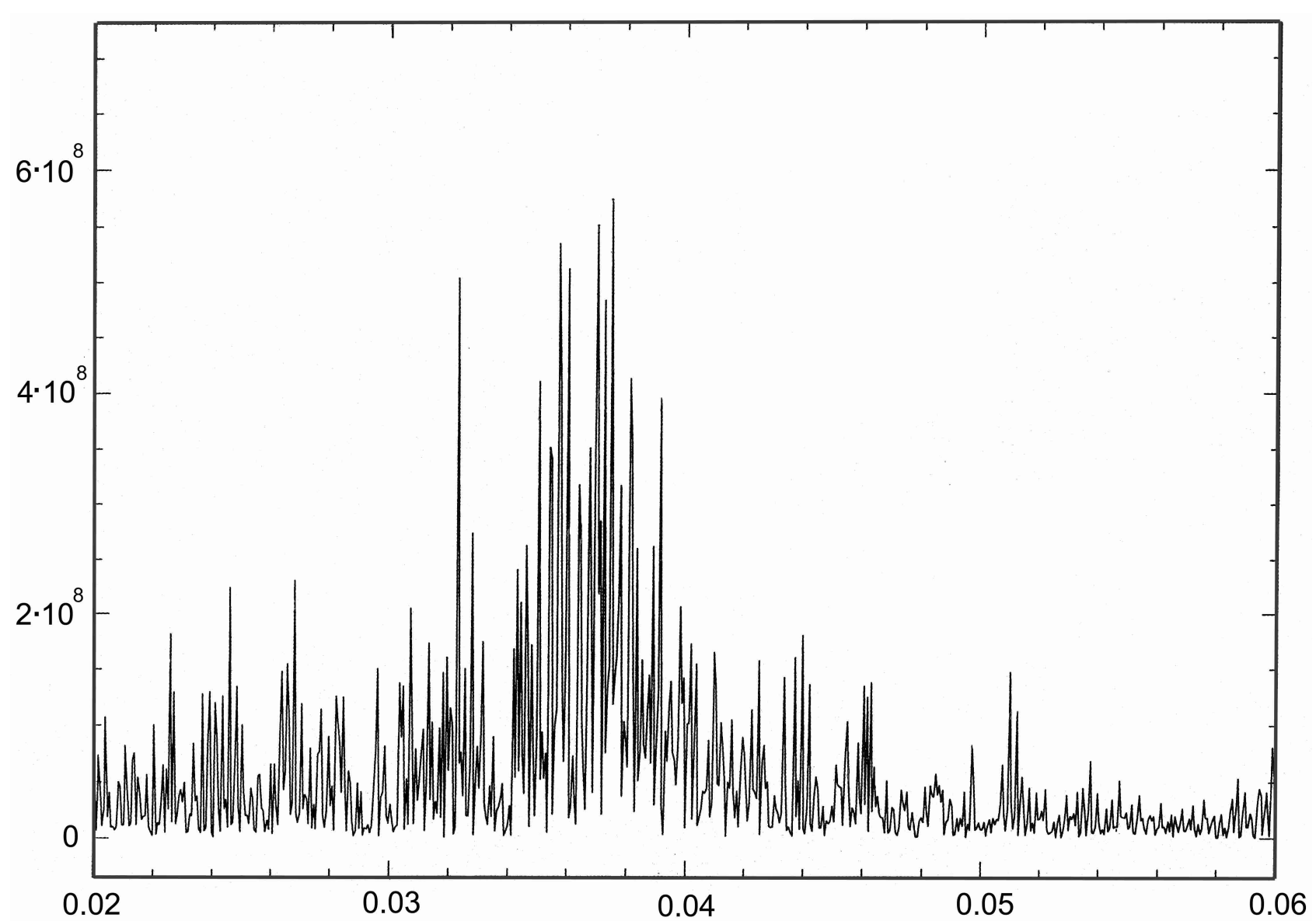

Fig. 3. The power spectrum of the same period as in Fig. 2, in abscissa frequencis 1/d.

In our data, the signal was neither less than 65 nor greater than 370 sfu. We use the Butterworth numerical filter (Stearns 1975) to cut off frequencies below $1 / 365 \mathrm{~d}$ and give in Fig. 2 the whole period analyzed (February 3, 1947 to November 9, 1998, or JD 2432258 to 2451 127), covering four solar cycles, 19 to 22 .

A preliminary study of rotation rate versus cycle, based on the material mentioned above, was already given by Mouradian et al. (2000). In order to determine the period of rotation we used a method of counting emission summits in a given lapse of time, around the maximum, intermediary and minimum epochs of solar activity. We found longer periods of rotation during the minima and shorter ones during the activity maxima. In the present paper, we perform the same task using the spectral analysis method.

\section{Coronal rotation rate}

Figure 3 shows the power spectrum of the $10.7 \mathrm{~cm}$ flux over the whole data for period intervals from 16.5 to $50 \mathrm{~d}$. We see an large group of frequencies spread around the Carrington Rotation period of $27.27 \mathrm{~d}$. It shows that coronal rotation is composed of multiple frequencies and therefore has to be studied in detail. We can distinguish two groups of frequencies, one between 0.034 and 0.040 and the second between 0.030 and $0.0341 / \mathrm{d}$. The first group is composed of well-defined frequencies, but the second one is more confused. Later on we shall see that they correspond respectively to active and quiet sun.

Various solar activity analyses have revealed a period of 150 to $160 \mathrm{~d}$ (Ichimoto et al. 1985; Oliver et al. 1998; the present data and others). To sample the $10.7 \mathrm{~cm}$ data we first chose a time lapse of $160 \mathrm{~d}$ and in order to reduce the noise, we considered three times this period, i.e. $480 \mathrm{~d}$ (approximately 18 solar rotations or $1.3 \mathrm{y}$ ). Consequently, the length of $51.7 \mathrm{y}$ was covered by 77 overlapping periods of $480 \mathrm{~d}$, shifted by $240 \mathrm{~d}$ steps. For each time interval of $480 \mathrm{~d}$, we computed the power spectrum using the Maximum Entropy Method (Press et al. 1990), which determines the frequency variation more accurately than other methods (Radoski et al. 1975). Each spectrum was normalized to its maximum, so that the frequencies occurring in low-activity periods were brought out too, thereby showing the rotation frequencies at any point in the cycle.

\section{Solar activity cycle and rotation rate}

We studied solar cycles 19, 20, 21 and 22 separately, focusing on the ten most active years (Fig. 4). For each cycle, a frame is given which is composed of 17 partially overlapping bins of power spectra of $480 \mathrm{~d}$, disposed in ordinate according to the dates of the observations. The $x$-axis shows the synodic rotation period. The contours represent the prominent maxima of the power spectra. For reference, we superimposed the radio flux variation in arbitrary units on each frame. " $M$ " and 
Table 1. Coronal rotation characteristics.

\begin{tabular}{|c|c|c|c|c|c|c|c|}
\hline \multirow[b]{2}{*}{ cycle } & \multicolumn{3}{|c|}{ maxima } & \multicolumn{2}{|c|}{ minima } & \multicolumn{2}{|c|}{ quiet sun } \\
\hline & date & $P^{\oplus} \mathrm{d}$ & $\Omega^{\star \circ} / \mathrm{d}$ & $P^{\oplus} \mathrm{d}$ & $\Omega^{\star \circ} / \mathrm{d}$ & $P^{\oplus} \mathrm{d}$ & $\Omega^{\star \circ} / \mathrm{d}$ \\
\hline 1 & 2 & 3 & 4 & 5 & 6 & 7 & 8 \\
\hline 19 & 1957 Jul. & 25.35 & 15.19 & 31 & 13 & 32 & 12.1 \\
\hline 20 & 1969 Jan. & 25.30 & 15.21 & 29 & 13 & 31 & 12.6 \\
\hline 21 & 1980 Jun. & 25.55 & 15.08 & 30 & 13 & 32 & 12.2 \\
\hline 22 & 1890 Jun. & 25.40 & 15.16 & 28 & 14 & 32 & 12.2 \\
\hline
\end{tabular}
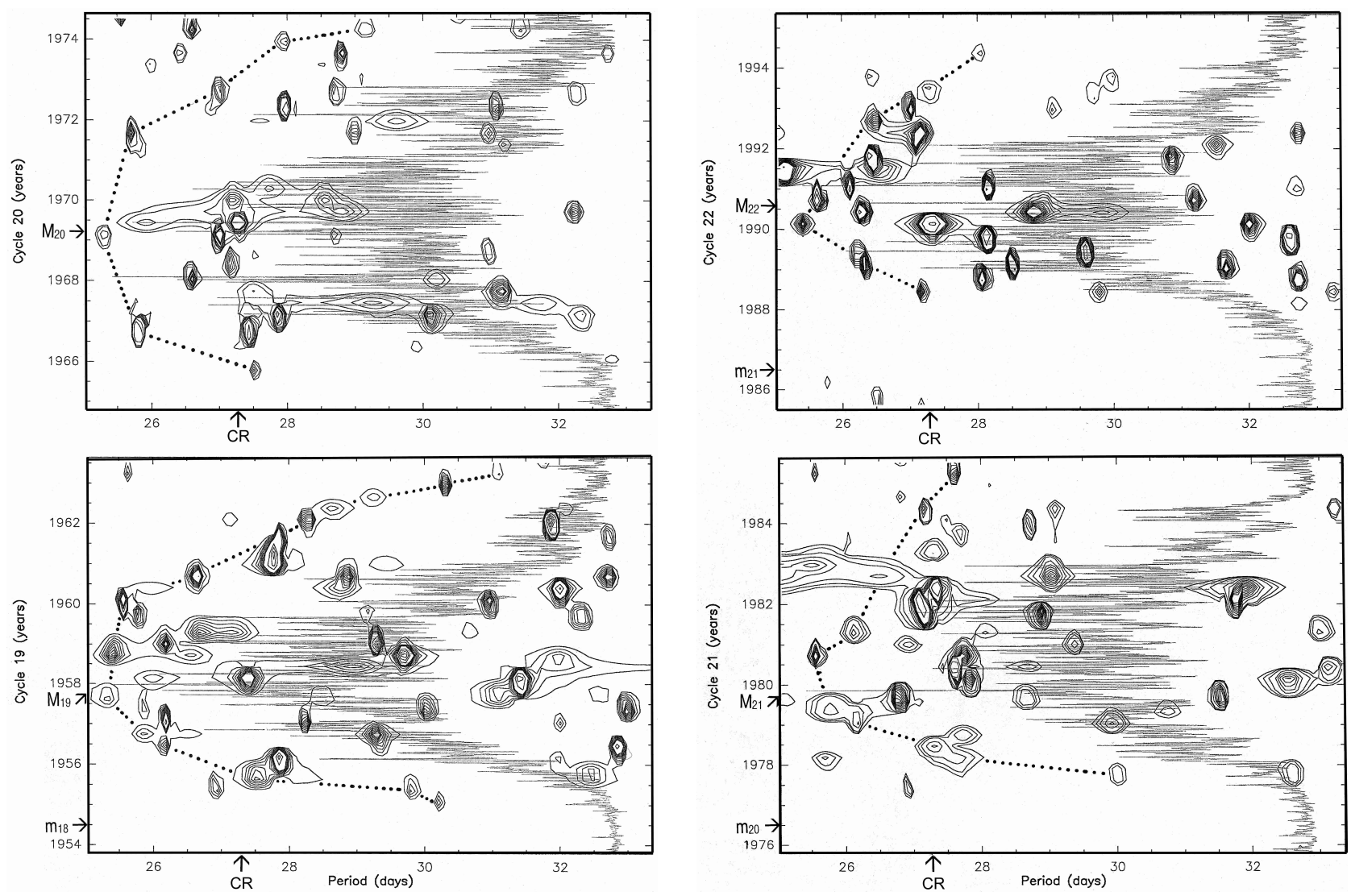

Fig. 4. Contour maps of power spectra for four cycles studied in the rotation period range from 25 to 33.5 days. $M$ and $m$ on the $y$-axis indicate the dates of cycle maximum and of minimum. CR on $x$-axis indicates the Carrington Rotation period in $1 / \mathrm{d}$. The dotted lines join the lower limit of rotation periods.

" $m$ " indicate respectively the Annual Mean Sunspot Numbers' (AMSN) maxima and minima (SGD 1997). The mark "CR" on the $x$-axis gives the position of the Carrington Rotation period.

\subsection{Activity and cycle}

Some points relating to the Fig. 4 can be emphasized:

- Solar rotation varies according to the solar cycle. For instance the most active cycle, number 19, clearly shows that the synodic rotation periods $\left(P^{\oplus}\right)$ are shorter at maximum activity than before and after:

- $P^{\oplus}=32.2 \mathrm{~d}$ in Jan. 1955 ( 2.5 y before cycle maximum),

- $P^{\oplus}=25.4 \mathrm{~d}$ in Jun. 1957 (cycle maximum),

- $P^{\oplus}=31.1 \mathrm{~d}$ in Feb. 1963 (5.6 y after maximum).
The time variation of the set of shortest rotation periods of each ordinates (bin) traces a C-shaped inferior limit of synodic rotation period, indicated by dotted lines. Frequencies with longer periods appear on the right hand side of this limit, untill periods of $33 \mathrm{~d}$. The other cycles (20,21 and 22) show the same general behavior. The right hand side end of the C-shaped limit (the highest rotation period) will be considered as the "minimum" of activity of the cycle. We consider periods higher than $31 \mathrm{~d}$ as "quiet sun".

- Table 1 displays the rotation characteristics resulting from the examination of the four cycles. The table gives the synodic rotation period $\left(P^{\oplus}\right)$ in days and the sidereal rotation rate $\left(\Omega^{\star}\right)$ in degrees per day. For each cycle figures concerning rotation maxima are provided in Cols. 3 and 4 , those for "minima" in Cols. 5 and 6 , and those for quiet sun in Cols. 7 and 8. 


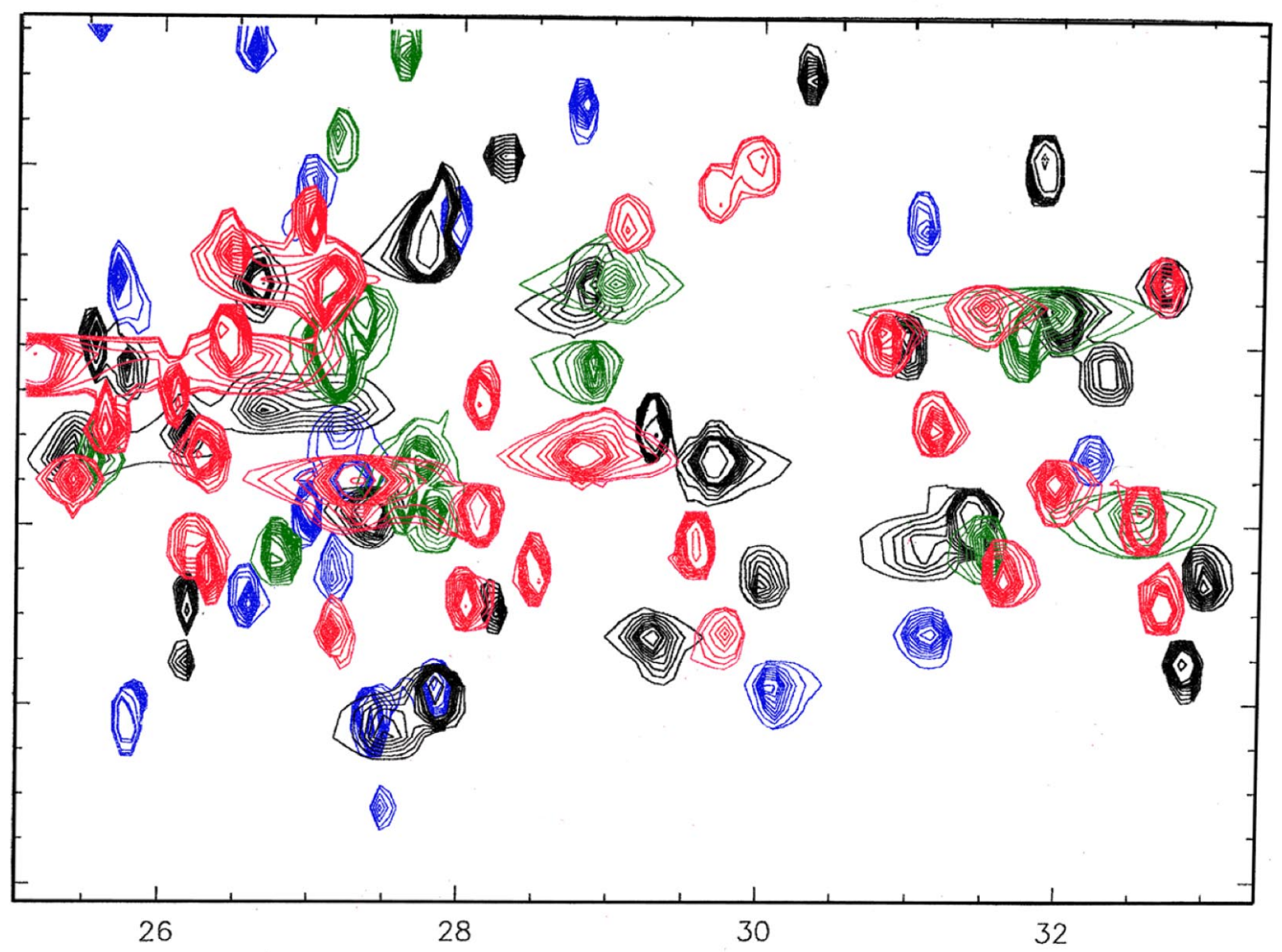

Fig. 5. Superimposition of power spectra peaks of frequencies of cycles 19 to 22 . Ordinate divisions are half-years. To distnguish the different cycles. (This figure is available in color in electronic form).

- No correlation can be established between the Monthly Mean Sunspot Number (MMSN) maximum of the cycle and its minimum of rotation period. However the four cycles have a wide variety of activity levels (Table 2, Col. 2). The maximum of cycle 20 is only $54 \%$ of the maximum of cycle 19 , whereas the rotation rate variation is only $0.2 \%$. We also note that coronal rotation period maximum and sunspot number maximum do not necessarily coincide in time (Tables 1 and 2). Column 3 of Table 2 gives the time difference in months between these maxima, if any.

- During the period of maximum of activity, there are broader frequency ranges, whereas in "minimum" this range becomes narrower.

- For long periods around activity minima, no outstanding frequencies are sufficiently revealed, because at minimum the active regions are generally few, small and short-lived. Under these conditions, the absence of information does not mean lack of preferential longitudes; moreover Fig. 3 shows a outstanding frequency at $P^{\oplus}=31 \mathrm{~d}$.

- The quiet sun is always present during the active period (frequencies $1 / 31$ to $1 / 33$ ) and does not show significant variation.
- Time variations of rotation periods (Table 3) are steeper in the increasing phase (average $0.8^{\circ} / \mathrm{d} / \mathrm{y}$ ) than in the decreasing phase $\left(-0.5^{\circ} / \mathrm{d} / \mathrm{y}\right)$ of the activity cycle.

- The accuracy of the choice of $480 \mathrm{~d}$ is subsequently justified by the extension of the $y$-axis of frequency peaks. It is evident that lower time resolution analysis hides important results.

- Not all the frames of Fig. 4 show frequency concentration around the Carringtion Rate. Keeping only the most prominent frequencies in the four frames of Fig. 4, we superimpose them and build Fig. 5. This shows frequencies around the Carrington Rotation period, in agreement with Fig. 3. We see around the activity maximum period (approximately 4 to 5 years) a richness of frequencies with values higher than the Carrington Rate.

- Figure 5 shows a fish-bone structure, composed of a set of three or four C-shaped layers of frequencies, making rotation rate variation obvious throughout the cycle. All cycles participate in each C-shaped structure, showing that this is a general characteristic of magnetic flux related to rotation rate. All fish-bone components show the same curvature, i.e. the same variation during the cycle. In Fig. 4, we again 
Table 2. Sunspot activity maxima.

\begin{tabular}{ccc}
\hline \hline date & AMSN & $\Delta T /$ month \\
1 & 2 & 3 \\
\hline 1957 Oct. & 190.2 & - \\
1969 Mar. & 105.9 & - \\
1979 Sep. & 155.4 & -9 \\
1989 Jun. & 157.2 & -12 \\
\hline
\end{tabular}

Table 3. Acceleration of rotation period in $\% / d / y$.

\begin{tabular}{ccc}
\hline \hline cycle & increasing phase & decreasing phase \\
\hline 19 & 0.9 & -0.8 \\
20 & 1.1 & -0.6 \\
21 & 0.8 & -0.3 \\
22 & 0.6 & -0.3 \\
\hline
\end{tabular}

find this fish-bone structure in each frame. Do these fishbones correspond to the rotation rate of different components of the solar atmosphere, or are they due to magnetic tubes rooted at different depths in the convective zone?

\subsection{Rotation and magnetic flux}

For frequencies in Fig. 4 we compute an activity index $R_{15}$, a 15 month mean of MMSN. The 15 month period is the closest odd number to $480 \mathrm{~d}$. Note that $R_{15}$ is very close to AMSN. In Fig. 6 we plot the sidereal rotation rate $\left(\Omega^{\star}\right)$ and the activity index $\left(R_{15}\right)$. Solid signs mark the frequencies of the Cshaped limits of the cycles, frequencies jointed by dotted lines in Fig. 4. The set of solid points (cycles 19, 21 and 22) can be approximated by an empirical equation:

$R_{15}=32 \times\left(\Omega^{\star}-13\right)^{2}+25$ for $\Omega^{\star} \geq 13^{\circ} / \mathrm{d}$.

The open signs show the frequency of the right hand side of the C-shape limits in Fig. 4. In the plane $\Omega^{\star}-R_{15}$ the frequency distribution also shows a lower limit of rotation rate, following the relation:

$R_{15}=75 \times\left(\Omega^{\star}-13\right)^{4}+25$ for $\Omega^{\star} \leq 13^{\circ} / \mathrm{d}$.

Curves (1) and (2) are shown in Fig. 6.

As far as cycle 20 (the weakest of our sample) is concerned, the two limiting curves (1) and (2) are valid with a translation of $+0.5^{\circ} / \mathrm{d}$. This shift indicates the necessity of a faster rotation to ensure magnetic field emergence. Using Eqs. (1) and (2) we can find a dependence of sunspot number on the rotation rate:

$$
\begin{aligned}
& 200 \geq R_{15} \geq 32 \times\left(\Omega^{\star}-\Omega_{0}^{\star}\right)^{2}+25 \text { for } \Omega^{\star} \geq \Omega_{0}^{\star} \\
& 200 \geq R_{15} \geq 75 \times\left(\Omega^{\star}-\Omega_{0}^{\star}\right)^{4}+25 \text { for } \Omega^{\star} \leq \Omega_{0}^{\star}
\end{aligned}
$$

here $\Omega_{0}^{\star}=13^{\circ} / \mathrm{d}$ for cycles 19,21 and 22 , and $\Omega_{0}^{\star}=13.5^{\circ} / \mathrm{d}$ for cycle 20 .

In order to express the activity index $\left(R_{15}\right)$ in units of total magnetic flux $\left(\Phi_{|B|}\right)$, we determine the following empirical relation, based on cycle 21 :

$\Phi_{|B|}=4 \times 10^{21} \times\left(R_{15}+50\right) \mathrm{Mx}$.

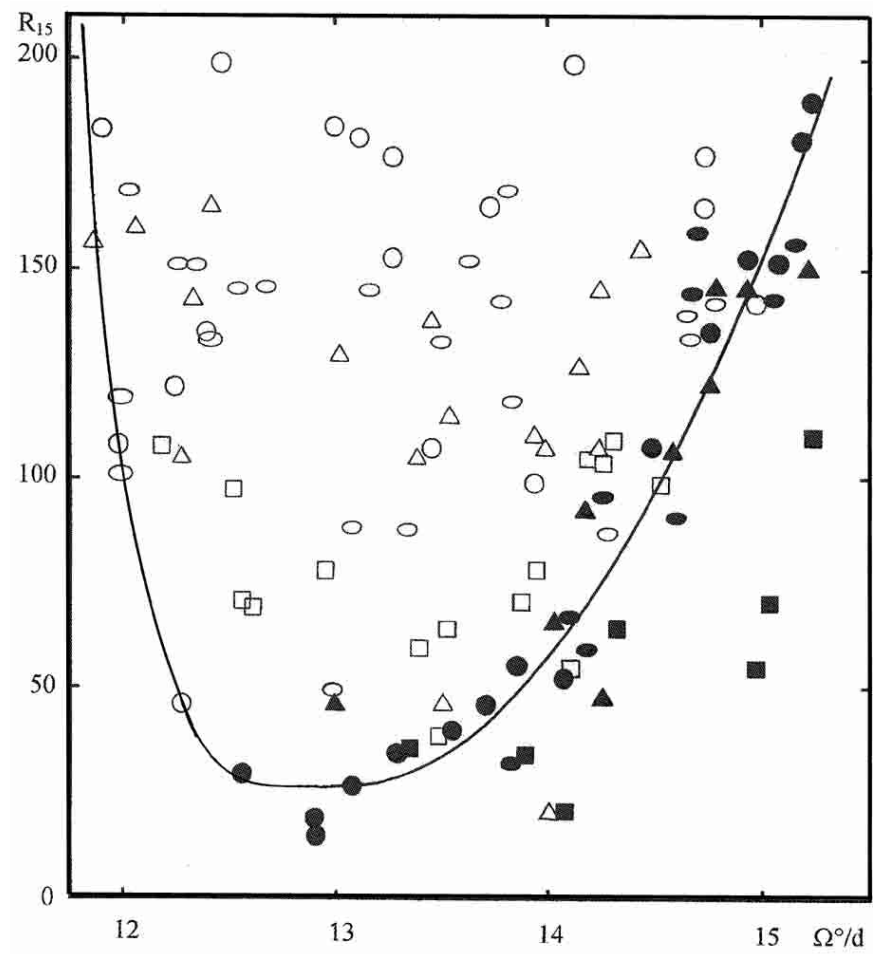

Fig. 6. Relationship between the sidereal rotation rate $\left(\Omega^{\star}\right)$ and solar activity $\left(R_{15}\right)$. Cycle 19 - circles, cycle 20 - squares, cycle 21 - triangles, cycle 22 - ellipse. Solid points are limit of rotation period, linked by dotted lines in Fig. 4. The curves draw the upper and lower rotation rates limits at a given activity index, accordingly to Eqs. (1) and (2).

Figure 6 indicates that, for a given rotation rate $\Omega^{\star}$, only activity fluxes equal to or greater than that given by Eqs. (1) and (2) can emerge. Thus, the most likely rotation rate for the emergence of the magnetic field is $\Omega_{0}^{\star}$. At high or low rates, only stronger magnetic flux emerges, the weak flux being scattered before it reaches the top of the convective zone.

\subsection{Hysteresis}

Following the time evolution of sidereal rotation of the C-shape limits for each cycle (solid points of Fig. 6), we may see that during the starting phase of the activity cycle, the rotation rate first grows rapidly, whereas later the magnetic flux grows rapidly untill maximum. This fact shows the important role of the Coriolis force in magnetic field emergence.

We note that cycles 20, 21 and 22 display hysteresis loops during their time evolution. No hysteresis was detected for cycle 19, the strongest one. Especially for cycles 21 and 22, the hysteresis shows that in the decreasing phase higher rotation is needed $\left(\Delta \Omega^{\star}=0.3^{\circ} / \mathrm{d}\right)$ for the emergence of the same magnetic flux than during the increasing phase.

\section{Conclusions}

In this paper we propose a new method for measuring rotation rate and clearly establish that: a) the active corona rotation rate varies with respect to the 11-y sunspot cycle, b) there is a relationship between rotation rate and magnetic flux, 
c) rotation grows first and then the magnetic field emerges, d) activity increases the rotation rate. The sidereal rotation rate of the active corona varies between $15.2^{\circ} / \mathrm{d}$ and $13^{\circ} / \mathrm{d}$ respectively, for activity maxima and "minima". Nevertheless $10.7 \mathrm{~cm}$ rates of maxima and of "minima" include the sunspot radiation rates. Lastly, active regions in the photosphere and in the corona averaged over the whole cycle reproduce the Carrington Rotation Rate. On the other hand, the frequencies over the whole observation time, given in Fig. 3, show different frequencies grouped round the Carrington Rate.

The rotation figures given in this paper have larger amplitudes than those of other authors. This is due to the high temporal resolution of the data and to the narrow windows used for computation of power spectra. Even if sunspots and $10.7 \mathrm{~cm}$ coronal flux data are disk observations, a direct comparison cannot be done. In case of sunspots, the measurements give differential rotation rates over long periods including cycles of activity. Data from analyses of $10.7 \mathrm{~cm}$ flux are frequencies distributed with respect to time, which cannot be averaged. According to Gilman \& Howard (1984) and to Gupta et al. (1999), at the minimum of activity, sunspot rotation is faster than during the rest of the cycle, whereas coronal rotation is slower at that time, as was pointed out for all coronal observations. This remains unexplained.

Antonucci \& Dodero (1977) showed the simultaneous presence of coronal structures with differential rotation and with rigid rotation in the same period. It is also well known that coronal holes show either rigid or differential rotation. This assertion is supported by the detection of Pivot Points (Mouradian et al. 1987), which designate regions of the solar photosphere in rigid rotation, alongside regions in differential rotation. Stenflo's (1977) sentence, “There is a coexistence on the sun of regions with rigid and differential-rotation properties" is here proved for the corona too. Observations revealed the simultaneous presence of a large number of rates, so that the smoothed differential rotation cannot be considert as a local rule.

Acknowledgements. The $10.7 \mathrm{~cm}$ Solar Flux data were provided by the National Research Council of Canada and the authors are in dept to Dr. K. Tapping. We are grateful for clarifying discussions to Dr. D. Heristchi. The authors thank the referee, Dr. H. Wöhl, for his valuable remarks and comments.

\section{References}

Antonucci, E., \& Dodero, A. 1977, Sol. Phys., 53, 179

Badalyan, O. G., \& Livshits, M. A. 1997, Ap\&SS, 252, 317

Balthasar, H., Vázquez, M., \& Wöhl, H. 1986, A\&A, 155, 87

Beck, G. 1999, Sol. Phys., 191, 47

Becker, U. 1955, Z. Astrohys., 37, 47

Benevolenskaya, E. E., Kosovichev, A. G., \& Scherrer, P. H. 2000, BAAS, 32, 815

Brouwer, M. P., \& Zwaan, C. 1990, Sol. Phys., 129, 221

Covington, A. E. 1969, JRASC, 63, 125
Fisher, R. R., \& Sime, D. G. 1984, ApJ, 287, 959

Gaizauskas, V., Harvey, K. L., Harvey, J. W., \& Zwaan, C. 1983, ApJ, 265,1056

Gilman, P. A., \& Howard, R. 1984, ApJ, 283, 385

Gupta, S. S., Siviraman, K. R., \& Howard, R. F. 1999, Sol. Phys., 188, 225

Hansen, R. T., Hansen, S. F., \& Loomis, H. G. 1969, Sol. Phys., 10, 135

Hathaway, D. H., \& Wilson, R. M. 1990, ApJ, 357, 271

Henze, W. Jr., \& Dupree, A. K. 1973, Sol. Phys., 33, 425

Hoeksema, J. T., \& Scherrer, P. H. 1987, ApJ, 318, 428

Howard, R. 1984, ARA\&A, 22, 131

Howard, R. F., Gilman, P. A., \& Gilman, P. I. 1984, ApJ., 283, 373

Howard, R. F., Gupta, S. S., \& Siviramann, K. R. 1999, Sol. Phys., 186, 25

Howard, R. F., Kichatinov, L. L., Bogart, R. S. \& Ribes, E. 1991, in Solar Interior and Atmosphere, ed. A. N. Cox, W. C. Livingston, $\&$ M. S. Matthews (The University of Arizona Press, Tucson), 748

Ichimoto, O., Kubota, J., Suzuki, M., Tohmura, I., \& Kurokawa, H. 1985, Nature, 316, 422

Kundu, M. R. 1965, Solar Radio Astronomy (Interscience Publ.), 152 Libbrecht, K. G., \& Morrow, C. A. 1991, in Solar Interior and Atmosphere, ed. A. N. Cox, W. C. Livingston, \& M. S. Matthews (The University of Arizona Press, Tucson), 479

Livingston, W. C., \& Duvall, T. L. 1979, Sol. Phys., 61, 219

Lustig, G. 1983, A\&A, 125, 355

Mordvinov, A. V., \& Plyusnina, L. A. 2000, Sol. Phys., 197, 1

Mouradian, Z., Bocchia, R., \& Botton, C. 2000, C. R. Acad. Sci. Paris, 1, Ser. IV, 353

Mouradian, Z., Martres, M. J., Soru-Escaut, I., \& Gesztelyi, L. 1987, A\&A, 183, 129

Oliver, R., Ballester, J. L., \& Baudin, F. 1998, Nature, 394, 552

Parker, G. D. 1986, Sol. Phys., 104, 333

Press, W. H., Flannery, B. P., Teukolsky, S. A., \& Vetterling, W. T. 1990, Numerical Recipes (Cambridge Univ. Press), 447

Pulkkinen, P., \& Touminen, I. 1998, A\&A, 332, 748

Rabin, D. M., DeVore, C. R., Sheeley, N. R. Jr., \& Harvey, K. L. 1991, in Solar Interior and Atmosphere, ed. A. N. Cox, W. C. Livingston, \& M. S. Matthews (The University of Arizona Press, Tucson), 781

Radoski, H. R., Fougere, P. F., \& Zawalick, E. 1975, J. Geophys. Res., 80, 619

Rybak, J. 1994, Sol. Phys., 152, 161

Schröter, E. H. 1985, Sol. Phys., 100, 141

SGD 1997, Solar-Geophys. Data, Nr. 640, Part 1

Stearns, S. D. 1975, Digital Signal Analysis, Hayden Book Company, Inc., 183

Stenflo, J. O. 1977, A\&A, 61, 797

Swarup, G., Kakinuma, T., Covington, A. E., et al. 1963, ApJ, 137, 1251

Tapping, K. F., \& DeTracey, B. 1990, Sol. Phys., 127, 321

Van Driel-Gesztelyi, L., van der Zalm, E. B. J., \& Zwaan, C. 1992, in The Solar Cycle, ASP Conf. Ser., 27, 89

Vats, H. O., Deshpande, M. R., Meyta, M., et al. 1999, Adv. Space Res., 24(2), 241

Vats, H. O., Cecatto, J. R., Meyta, M., Sawant, H. S., \& Neri, A. C. F. 2001, ApJ, 548, L87

Weber, M. A., Action, L. W., Alexander, D., Kubo, S., \& Hara, H. 1999, Sol. Phys., 189, 271

Woodard, M. F., \& Libbrecht, K. G. 1993, Science, 260, 1778 